Review Article

\title{
Role of Long Noncoding RNAs in Parkinson's Disease: Putative Biomarkers and Therapeutic Targets
}

\author{
Qiankun Lv, Ziyu Wang, Zhen Zhong, and Wei Huang $(D$ \\ Department of Neurology, The Second Affiliated Hospital of Nanchang University, Nanchang, China \\ Correspondence should be addressed to Wei Huang; 13677080198@163.com
}

Received 4 March 2020; Revised 7 May 2020; Accepted 21 May 2020; Published 12 June 2020

Academic Editor: Giuseppina Martella

Copyright ( 2020 Qiankun Lv et al. This is an open access article distributed under the Creative Commons Attribution License, which permits unrestricted use, distribution, and reproduction in any medium, provided the original work is properly cited.

\begin{abstract}
Parkinson's disease (PD) is a neurodegenerative disease characterized by bradykinesia, rigidity, and tremor. Age is the main risk factor. Long noncoding RNAs (lncRNAs) are novel RNA molecules of more than 200 nucleotides in length. They may be involved in the regulation of many pathological processes of PD. PD has a variety of pathophysiological mechanisms, including alphasynuclein aggregate, mitochondrial dysfunction, oxidative stress, calcium homeostasis, axonal transport, and neuroinflammation. Among these, the impacts of lncRNAs on the pathogenesis and progression of PD need to be highlighted. lncRNAs may serve as putative biomarkers and therapeutic targets for the early diagnosis of PD. This study aimed to investigate the role of lncRNAs in various pathological processes of PD and the specific lncRNAs that might be used as putative diagnostic biomarkers and therapeutic targets of PD.
\end{abstract}

\section{Introduction}

Parkinson's disease (PD) is a common neurodegenerative disease, second only to Alzheimer's disease (AD) [1]. It is characterized by bradykinesia, rigidity, and tremor, affecting $2 \%-3 \%$ of the population aged more than 65 years [2]. Various epidemiological and experimental studies have shown that old age, environmental risk factors, and genetic factors are related to the pathogenesis of PD. Old age is the leading risk factor for PD [3]. Although PD is rare before 50 years of age, its incidence increases five- to tenfold from the sixth to the ninth decade of life [4].

Recent studies focus on the pathogenesis of PD at both the microscopic biological and macroscopic anatomical levels to find an effective therapy [5]. PD has a variety of pathophysiological mechanisms, including alpha-synuclein aggregate, mitochondrial dysfunction, oxidative stress, calcium homeostasis, axonal transport, and neuroinflammation [6]. The combined action of these mechanisms causes the death of dopaminergic neurons in the substantia nigra, promoting the development of PD.

Despite the noteworthy advances in understanding the etiology and the high-throughput drug screening methods for small molecules, remarkable developments in disease modeling, and improvements in analytical technologies, no therapies are available to prevent the disease from getting worse [7]. More importantly, the current diagnosis of PD depends mainly on the clinical symptoms, and the diagnosis can be confirmed only by autopsy [8]. Therefore, further understanding the molecular mechanism of PD and proposing effective therapeutic targets and treatment programs remain high priorities.

Long noncoding RNA (lncRNA) is a new potential biomarker with biological functions [9]. It has a broad clinical application prospect. Recent studies on $\operatorname{lncRNAs}$ have attempted to reveal the mystery of PD. IncRNAs are novel RNA molecules of more than 200 nucleotides in length. They are located in the nucleus or cytoplasm. They play potential roles in regulating the expression levels of protein-coding genes through epigenetic regulation, transcriptional regulation, and posttranslational control, although they hardly or do not encode proteins themselves $[10,11]$.

lncRNAs function by interacting with three kinds of biomolecules: DNA, RNA, and protein [12]. They form binary and even ternary complexes. Hence, looking for 
targets interfering with their interactions can help in drug discovery. In the nucleus, they can specifically induce gene silencing on the same or another chromosome by recruiting chromosomal remodeling complexes to specific chromosomal locations [10]. In the cytoplasm, they can serve as competing endogenous RNAs (ceRNAs) to modulate miRNA expression [13-15]. They can also control transcriptional activity by directly or indirectly targeting mRNAs [16].

lncRNAs are highly enriched and expressed in the central nervous system (CNS) [17]. They play a role in neural development and brain evolution through histone modification, transcription cofactors, mRNA decay, and alternative splicing [18], thus mediating behavior and cognition $[18,19]$.

The abnormal expression of lncRNAs is closely associated with several human neurological diseases, including PD, AD, Huntington's disease, and schizophrenia [20-23]. Importantly, lncRNA expression changes during aging, thus serving as the major risk factor for the development of PD $[24,25]$. Although the research on the role of lncRNAs in PD is very limited at present, the potential regulatory mechanism should not be ignored. In conclusion, lncRNA, as an important endogenous regulatory mechanism in a human body, is expected to become a new therapeutic target to prevent PD from getting worse. This study provided brief, but focused insights into the role of lncRNAs in regulating multilevel activities in PD (Figure 1).

\section{Aberrant Expression of lncRNAs in PD}

Existing studies have confirmed that lncRNAs are highly expressed in various parts of both the CNS and the brain $[26,27]$. Several studies have shown the involvement of aberrant lncRNAs in the pathological process of PD (Table 1 and 2). lncRNAs were detected in brain tissues (the cingulate gyrus) of patients with PD. Five significantly differentially expressed lncRNAs were found, including significantly upregulated expression of H19 upstream conserved 1 and 2 and significantly downregulated expression of long intergenic noncoding RNA-p21 (lincRNA-p21), metastasis-associated lung adenocarcinoma transcript 1 (MALAT1), small nucleolar RNA host gene 1 (SNHG1), and trophoblastderived noncoding RNA (TncRNA) [48]. Patients with early PD show Lewy-related pathology only in brain stem regions. Subsequently, the cingulate gyrus is affected. These significantly differentially expressed lncRNAs are expressed in the cingulate gyrus of patients, indicating that the disorder of lncRNAs may occur in the early stage of PD and has the potential to be used as a biomarker in the early stage of the disease. A recent study found that PD-related genes associated with lncRNAs decreased in the substantia nigra and cerebellum of patients, which was consistent with the results obtained in peripheral blood monocytes [47]. Most of them were detected in the cerebrospinal fluid- (CSF-) derived exosomes, providing the basis for lncRNAs as a potential biomarker of PD. IncRNAs in the CSF of patients with PD have a higher frequency compared with controls, corroborating previous reports that various lncRNAs performed essential functions in the regulation of progression of $\mathrm{PD}$ [50]. CSF is close to the main site of PD pathology and is an optimal source of biomarkers for neurodegenerative disorders because of the lack of a barrier between CSF and the brain [57]. IncRNAs in CSF have the potential as a biomarker for PD. In addition, the change in lncRNAs may be one of the patient's symptom severity indexes. In a comparative study, deep brain stimulation could effectively improve the symptoms of patients with PD and change the blood leukocyte cells of 663 lncRNAs [20]. Similarly, PDrelated lncRNAs were also found in different PD animal and cell models. The SH-SY5Y cell line stimulated with alphasynuclein produced 53 upregulated lncRNAs and 69 downregulated lncRNAs compared with the control group [5]. The specific downregulation of AS Uchl1 in iMN9D cell lines stimulated with 1-methyl-4-phenylpyridinium (MPP+) was also verified [28]. Further, 279/164 upregulated lncRNAs and 477/177 downregulated lncRNAs were found in Th-SNCA*A30P*A53T transgenic mice and alpha-synuclein transgenic mice $[53,55]$, respectively. In Nrf2 knockout mice, 74 upregulated lncRNAs and 160 downregulated $\operatorname{lncRNAs}$ were found [54]. Differential lncRNA expression in conventional nontransgenic PD models has also been studied. A total of $512 \operatorname{lncRNAs}$ related to PD have been identified in a 6-hydroxydopamine (6-OHDA) rat PD model, among which 54 are known lncRNAs [56].

lncRNAs detected in the aforementioned studies were different because of different sampling locations and detection techniques, besides differences between individuals. They play an important role in the pathological process of $\mathrm{PD}$ and may serve as a potential diagnostic marker and therapeutic target for PD. Furthermore, they may also be used to quantify the efficacy of medication and surgery in patients. However, obtaining a large number of multicenter databases to quantify lncRNA changes in PD is difficult due to the high cost of sequencing technology. However, with the progress of science and technology, lncRNAs have a great potential as a diagnostic marker and therapeutic target for PD.

\section{Association of IncRNAs with PD- Linked Genes}

Currently, six widely recognized sites, including SNCA (alpha-synuclein), Parkin (PARK2), PINK1 (PARK6), DJ-1 (PARK7), LRRK2 (PARK8), and ATP13A2 (PARK9), can cause hereditary single-gene PD [27]. As early as in 2016, the expression of lncRNA AC079630 and uc001lva.4 (close to the LRRK2 gene locus) was detected in the CSF of patients with PD [50]. In addition, lncRNA HOX Transcript Antisense RNA (HOTAIR) also regulated the expression of LRRK2 [30]. The microtubule-associated protein tau (MAPT) gene, which encodes tau protein, has also been identified as a susceptibility gene in PD [58]. High expression levels of MAPT may lead to an increase in the prevalence of neurodegenerative diseases [59]; the methylation of MAPT promoter is particularly related to PD [60]. In the pathology of PD, MAPT-AS1 can control the progression of the disease by inhibiting the methylation of the 


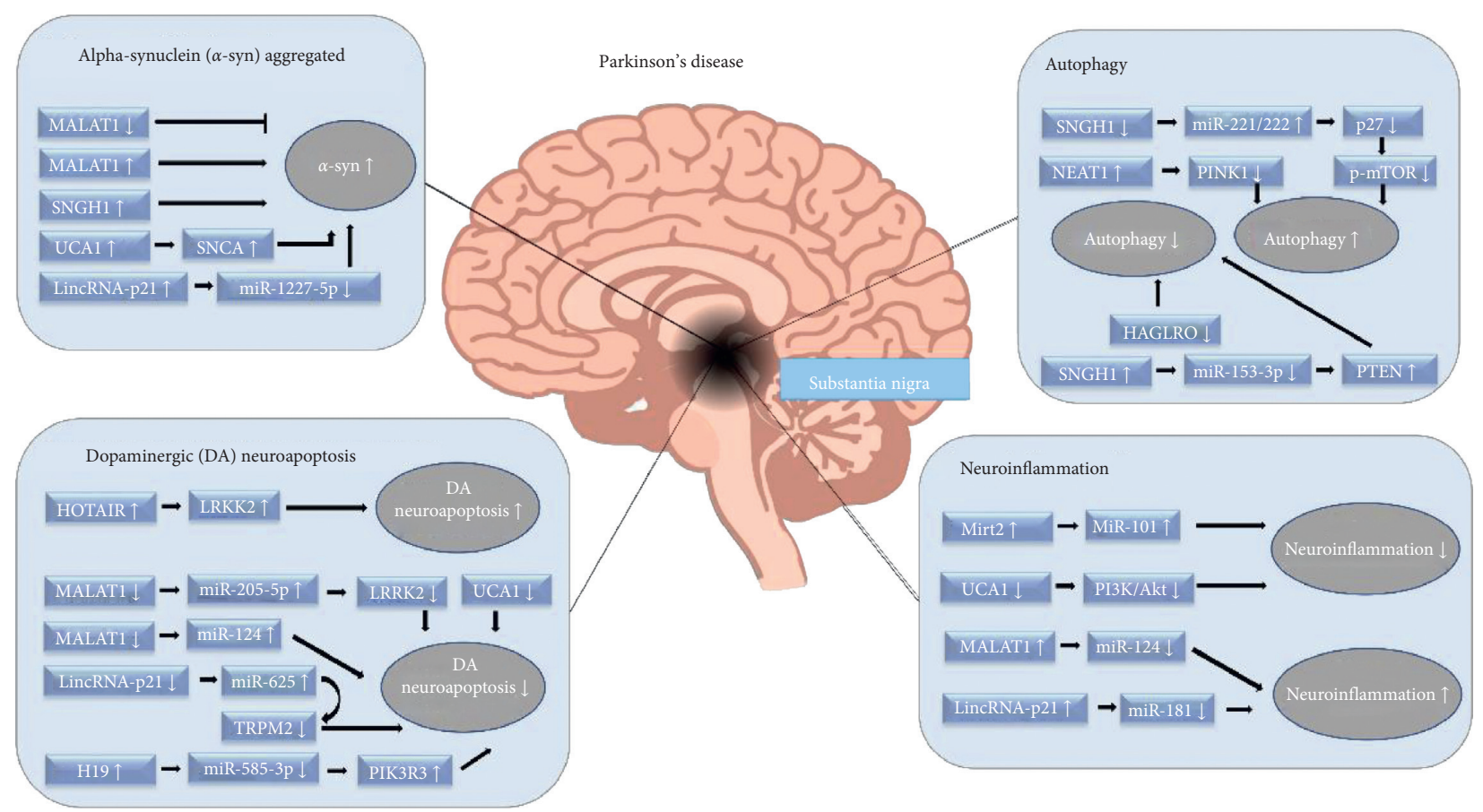

FIGURE 1: Multiple functions of long noncoding RNAs (lncRNAs) in pathological changes of Parkinson's disease.

MAPT promoter [35]. A recent study reported a number of lncRNAs encompassing transcriptional units in proximity to PD-linked protein-coding genes, including SNCA, LRRK2, PINK1, DJ-1, UCH-L1, MAPT, and GBA1. Also, a correlation was found between the expression profile of each lncRNA and its adjacent coding genes. This study indicated that these lncRNAs might be involved in the pathogenesis of the disease by regulating their adjacent PDrelated bases, thus having the potential to be used as biological diagnostic markers and therapeutic targets for PD [47].

\section{Roles of lncRNAs in PD Pathophysiology}

4.1. Aggregation of IncRNAs and Alpha-Synuclein in PD. Alpha-synuclein is a protein closely related to neurodegenerative diseases. It is an important part of the Lewy body, and its abnormal aggregation is associated with PD, Lewy body dementia, and multisystem atrophy [61]. It causes abnormal deposition of proteins in the cells of patients [62]. Hence, targeting alpha-synuclein can be a potential therapeutic target for PD [63]. Further, understanding the role of alpha-synuclein is important for understanding PD. The aberrant soluble oligomeric conformations of alpha-synuclein contribute to neuronal death and cellular homeostasis disruption. Multiple lncRNAs are clearly involved in this process. Microarray expression profiling was performed in control nontransgenic and human alpha-synuclein transgenic mice by stimulating alpha-synuclein. The expression of 341 lncRNAs in the transgenic mice was significantly different from that in the control mice [55]. This study proved that $\operatorname{lncRNAs}$ were involved in the pathological process of alpha-synuclein-induced PD. The same was the case in in vitro cell models. After treating SH-SY5Y cell lines with alpha-synuclein oligomers, the changes in lncRNAs were analyzed using a microarray. The results showed noteworthy changes in a series of lncRNAs, including G046036, G030771, AC009365.4, RPS14P3, CTB-11I22.1, and G007549 [5]. This study verified the involvement of lncRNAs in the pathological process of alpha-synucleininduced PD at the cellular level in vitro. lncRNA MALAT1, also known as NEAT2, was highly expressed in neurons and regulated a number of genes involved in dendritic and synaptic development $[64,65]$. The overexpression of MALAT1 upregulated the expression of alpha-synuclein in the brains of mice with PD and vice versa [32]. In addition, the specific overexpression of SNHG1 in the brain of mice also led to the aggregation of alpha-synuclein [42]. The highly expressed lincRNA-p21 aggravated the influence of alpha-synuclein on cells through sponging miR-1277-5p [40].

Three therapeutic views exist on the role of alpha-synuclein in PD: reducing the expression of SNCA by directly silencing or inhibiting its promoter expression, activating autophagy or proteasome to increase protein clearance, and reducing posttranslation-based modification [66]. lncRNAs are closely related to alpha-synuclein, and targeting these lncRNAs to act on alpha-synuclein may serve as a fourth therapeutic viewpoint.

4.2. IncRNAs and Autophagy in PD. A dynamic balance exists in the expression of alpha-synuclein in normal individuals. The balance is maintained by the actions of the ubiquitin-proteasome system and the lysosomal autophagy system (LAS). The LAS is more important than the 
TABLe 1: LncRNAs: their mechanism of action in Parkinson' disease.

\begin{tabular}{|c|c|c|c|c|}
\hline lncRNA & Tissue/model & Regulation & Pathway targeted by the lncRNAs & References \\
\hline AS UCHL1 & $\begin{array}{l}\text { (1) MN9D cells treated with MPP+ } \\
\text { (2) DA neurons from PD model treated } \\
\text { with MPP+ }\end{array}$ & Down & $\begin{array}{l}\text { AS Uchl1 RNA, as a component of Nurr1-dependent } \\
\text { gene network and target of cellular stress, extended the } \\
\text { understanding on the role of antisense transcription in } \\
\text { the brain }\end{array}$ & {$[28]$} \\
\hline HAGLROS & $\begin{array}{l}\text { MPTP-induced PD mice and SH-SY5Y } \\
\text { cells treated with MPP+ }\end{array}$ & Up & $\begin{array}{l}\text { Suppression of HAGLRO decreased apoptosis and } \\
\text { autophagy in both in vivo and in vitro PD models } \\
\text { HAGLRO negatively regulated miR-100 expression } \\
\text { Suppression of HAGLROS alleviated MPP(+)- } \\
\text { intoxicated SH-SY5Y cell injury by activating PI3K/ } \\
\text { AKT/mTOR pathway }\end{array}$ & {$[29]$} \\
\hline
\end{tabular}

\section{AKT/mTOR pathway}

With HOTAIR overexpression in SH-SY5Y cells, the expression of LRRK2 increased compared with that in

HOTAIR SH-SY5Y cells treated with MPP+ $\mathrm{Up}$ the control

HOTAIR knockdown provided protection against $\mathrm{MPP}(+)$-induced DA neuronal apoptosis by repressing caspase 3 activity

MPTP-induced PD mice and SH-SY5Y cells treated with MPP+

MPTP-induced PD mice and SH-SY5Y cells treated with MPP+

MALAT1

MPTP-induced PD mice and MN9D cells treated with MPP+

MPTP-induced PD mice and SH-SY5Y cells treated with MPP+

MALAT1 knockdown attenuated MPTP-induced apoptosis of DA neurons in MPTP-induced PD mouse

Up

MALAT1 interacted with miR-124 to negatively regulate

MALAT1 was associated with a-synuclein, leading to the increased stability of a-synuclein and its expression MALAT1/miR-205-5p axis regulates MPP(+)-induced apoptosis in MN9D cells by targeting LRRK2

MALAT1 knockdown attenuated MPP(+)-induced apoptosis of DA neurons in SH-SY5Y cells

Up MALAT1 regulates DAPK1 expression by targeting miR-

MAPT-AS1 and DNMT1 have been identified as Down potential epigenetic regulators of MAPT expression in

Mirt2 exhibited anti-inflammatory properties through

Down

SY5Y cells treated with TNF- $\alpha$

Mirt2 blocked TNF $\alpha$-triggered NF- $\kappa$ B/p38MAPK

NEAT1 knockdown promoted cell viability and

MPTP-induced PD mice and SH-SY5Y cells treated with MPP+

Up Downregulation of NEAT1 also decreased the ratio of

$\mathrm{Bax} / \mathrm{Bcl}-2$, the activity of caspase-3, as well as the

NEAT1

MPTP-induced PD mice and SH-SY5Y cells treated with MPP+

NEAT1 positively regulated the protein level of PINK1

through inhibition of PINK1 protein degradation

NEAT1 knockdown could effectively suppress MPTPinduced autophagy that alleviated dopaminergic

lincRNA-p21 regulated MPP(+)-induced neuronal

SH-SY5Y cells treated with MPP+

lincRNA-

$\mathrm{p} 21$
MPTP-induced PD mice and SH-SY5Y cells treated with MPP+

MPTP-induced PD mice and SH-SY5Y cells treated with a CM transfer system were used to determine the impact of LPS-treated BV2 cells
Up injury by sponging miR-625 and upregulating TRPM2 in SH-SY5Y cells

lincRNA-p21 sponged miR-1277-5p and indirectly

Up increased the expression of $\alpha$-synuclein to suppress

viability and activate apoptosis in SH-SY5Y cells p53/lincRNA-p21, together with miR-181/PKC- $\delta$, formed a double-negative feedback loop that facilitated sustained microglial activation and the deterioration of model its expression 124-3p PD miR-101 suppression pathway suppressed cell apoptosis expression of $\alpha$-synuclein neuronal injury neurodegeneration 
TABLE 1: Continued.

\begin{tabular}{|c|c|c|c|c|}
\hline $\operatorname{lncRNA}$ & Tissue/model & Regulation & Pathway targeted by the lncRNAs & References \\
\hline \multirow[t]{3}{*}{ SNHG1 } & $\begin{array}{l}\text { MPTP-induced PD mice and SH-SY5Y } \\
\text { cells treated with MPP+ }\end{array}$ & Up & $\begin{array}{l}\text { SNHG1 could directly bind to miR-15-5p and repress } \\
\text { miR-15-5p expression } \\
\text { Upregulation of miR-15b-5p alleviated } \alpha \text {-synuclein } \\
\text { aggregation and apoptosis by targeting SIAH1 } \\
\text { SNHG1 knockdown inhibited } \alpha \text {-synuclein aggregation } \\
\text { and } \alpha \text {-synuclein-induced apoptosis }\end{array}$ & {$[42]$} \\
\hline & MN9D cells treated with MPP+ & Up & $\begin{array}{l}\text { SNHG1 could competitively bind to the miR-221/222 } \\
\text { cluster and indirectly regulate the expression of p27/ } \\
\text { mTOR }\end{array}$ & {$[43]$} \\
\hline & SH-SY5Y cells treated with MPP+ & Up & $\begin{array}{l}\text { SNHG1 overexpression lowered viability and enhanced } \\
\text { apoptosis in MPP(+)-treated SH-SY5Y cells. }\end{array}$ & {$[44]$} \\
\hline H19 & $\begin{array}{l}\text { MPTP-induced PD mice and human } \\
\text { neuroblastoma cells treated with MPP+ }\end{array}$ & Down & $\begin{array}{c}\text { H19 attenuates apoptosis in MPTP-induced Parkinson's } \\
\text { disease } \\
\text { H19/miR-585-3p axis regulates MPP(+)-induced } \\
\text { apoptosis in human neuroblastoma cells cells by } \\
\text { targeting PIK3R3 }\end{array}$ & {$[45]$} \\
\hline UCA1 & 6-OHDA-induced PD rat & Up & $\begin{array}{l}\text { Downregulation of lncRNA UCA1 ameliorates the } \\
\text { damage of dopaminergic neurons, reduces oxidative } \\
\text { stress and inflammation in PD rats } \\
\text { Downregulation of lncRNA UCA1 inhibits the PI3K/Akt } \\
\text { signaling pathway. }\end{array}$ & {$[46]$} \\
\hline
\end{tabular}

ubiquitinproteasome system in mediating alpha-synuclein degradation in neurons [67]. Age is the biggest risk factor for PD [68]; some toxins such as MPTP may induce the disease symptoms. The reason may be the deterioration of the functions of these two systems with aging [69]. Coincidentally, lncRNA expression also changes with aging. Hence, lncRNAs participate in disease pathology through autophagy in PD. In LAS, chaperone-mediated autophagy and macroautophagy participate in the degradation of alphasynuclein $[67,70]$; lncRNAs also play an important role. The SNCA gene, a susceptibility gene in sporadic PD [71], has been repeatedly reported in recent studies on PD [72-74]. It has the specific function of encoding alpha-synuclein. Its point mutation disrupts the cell homeostasis of dopaminergic neurons and leads to disorders of autophagy, resulting in the abnormal deposition of alpha-synuclein protein in the cytoplasm and further promoting the progression of the disease [75]. IncRNA-UCA1 can upregulate the expression of SNCA to promote the progression of PD [76]. In addition, lncRNA NEAT1 (nuclear paraspeckle assembly transcript 1) has been shown to promote autophagy of dopaminergic neurons by stabilizing PINK1 protein in both in vivo and in vitro models of $\mathrm{PD}$, thereby alleviating the damage to dopaminergic neurons $[38,76]$. The downregulation of SNHG1 promotes the autophagy of dopamine neurons through mir-221/222/p27/mTOR, thus slowing down apoptosis [43]. The overexpression of SNHG1 attenuates autophagy by regulating PTEN/AKT/mTOR signaling pathway in SH-SY5Y cells via sponging miR-153$3 p$ [44].

In conclusion, lncRNAs can be targeted to restore and strengthen the cell homeostasis of patients, maintain the balance of the autophagy system, and further eliminate alpha-synuclein as a potential treatment for PD.
4.3. IncRNAs and Apoptosis of Dopaminergic Neurons in PD. The apoptosis of dopaminergic neurons is the characteristic pathological manifestation of PD. Various lncRNAs play different roles in this process. IncRNA HOTAIR was highly expressed in the MPTP-induced PD mouse model and MPP(+)-induced PD cell model. HOTAIR specifically improved the stability of LRRK2 mRNA (LRRK2 mutations are widely recognized as the most common cause of dominant PD, and LRRK2 is one of the risk factors for PD [77]) and upregulated its expression to promote the apoptosis of dopaminergic neurons. Knocking down HOTAIR would inhibit the apoptosis of dopaminergic neurons by reducing the activity of caspase-3 [30]. lncRNA MALAT1 is highly expressed in PD as a ceRNA to regulate miRNA expression. The knockout of MALAT1 in mice inhibited the MPTPinduced apoptosis of dopaminergic neurons by upregulating miR-124 [31, 34]. MALAT1/miR-205-5p axis regulates MPP(+)-induced apoptosis by targeting LRRK2 [33]. Similarly, the knockout of SNHG1 inhibited the MPTP-induced apoptosis of dopaminergic neurons in mice with PD by reducing alpha-synuclein-induced cytotoxicity [42], and $\mathrm{H} 19$ attenuates apoptosis through regulating miR-585-3p/ PIK3R3 [45]. The downregulation of UCA1 ameliorates the apoptosis of dopaminergic neurons. [46]. Suppression of HAGLRO can also decrease apoptosis and autophagy in both in vivo and in vitro PD models [29].

Slowing down the progression of the disease by targeting the regulation of these apoptosis-related lncRNAs is a promising therapeutic option.

4.4. IncRNAs and Neuroinflammation in PD. Neuroinflammation is regarded as one of the most common contributors to PD [78]. Increasing evidence suggests that 


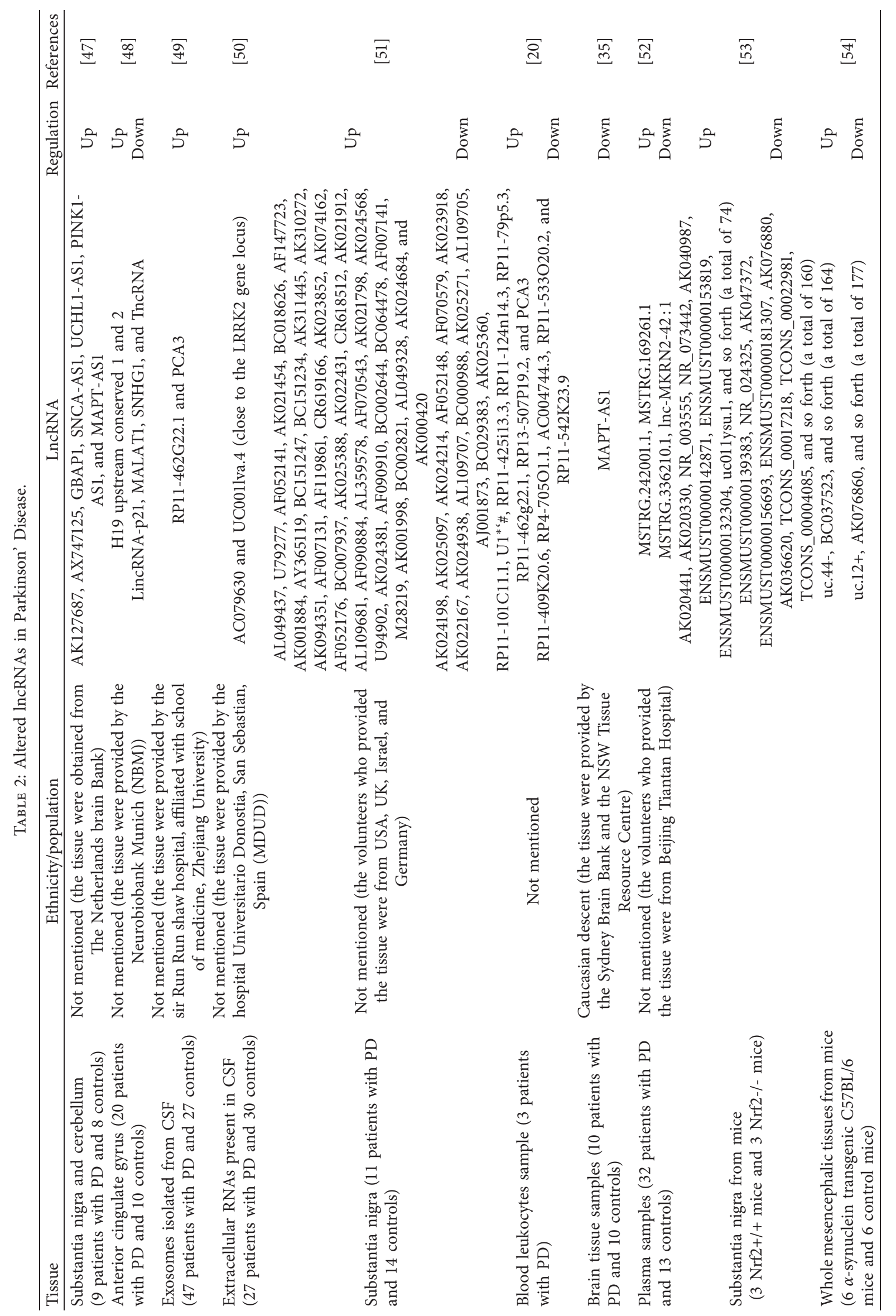




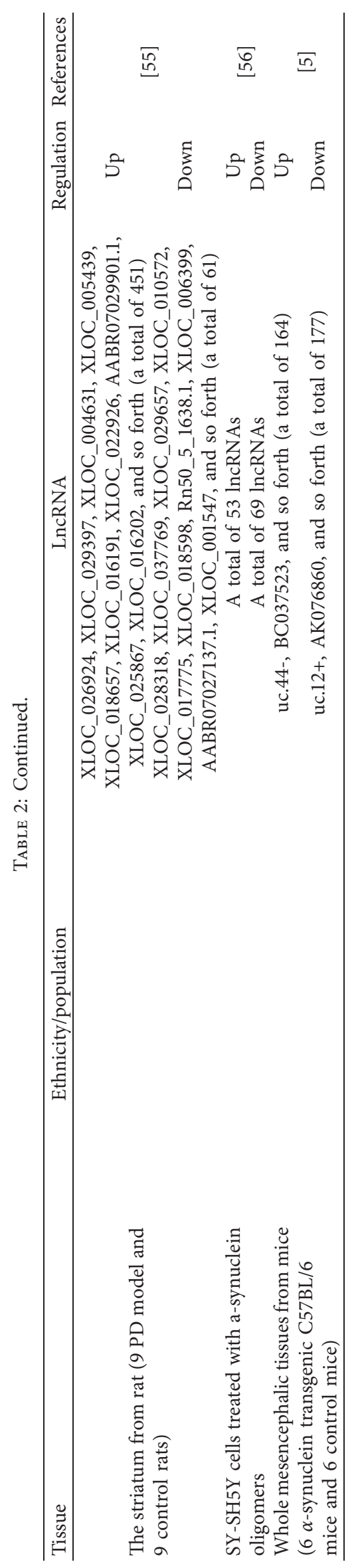


inflammation may serve as a crucial player in the death of dopaminergic neurons [79]. Existing studies demonstrated that lncRNAs were responsible for the differentiation of immune cells and corresponding immune response in mammals. When the innate immune system and inflammatory signals are overactivated, a large number of free radicals and proinflammatory cytokines are produced, leading to inflammatory cascade and neurodegeneration, which may be one of the molecular mechanisms of lncRNA involvement in PD pathology [80]. microRNA-124 reduced neuroinflammation in PD, while MALAT1 promoted a neuroinflammatory response in $\mathrm{PD}$ through sponging miR124 , leading to the secretion of a large number of proinflammatory factors and promoting the progression of PD [31]. IncRNA myocardial infarction associated transcript 2 (Mirt2) is considered to be a negative feedback medium for the excessive inflammatory response, indicating that Mirt2 can inhibit excessive inflammation [81]. In PD, Mirt2 can sponge $\mathrm{miR}-101$ to reduce the inflammatory response in neuropathology [36]. lincRNA-p21 (3100nt) is located on chromosome six. It is involved in cell proliferation, metabolism, and reprogramming and regarded as a potential diagnostic marker in various diseases [82]. In PD, lincRNAp21 aggravates the inflammatory response. As stated earlier, the highly expressed lincRNA-p21 inhibits the activity of dopaminergic neurons through sponging miR-1277-5p, thus aggravating the influence of alpha-synuclein on cells [40]. Further studies have shown that lincRNA-p21, as a ceRNA, constitutes an lncRNA-miRNA-mRNA regulatory network that indirectly acts on mRNA to exert its effect. For example, specifically knocking down lincRNA-p21 in PD reduces $\mathrm{MPP}(+)$-induced neuronal damage by regulating the mir625/TRPM2 axis [39]. Downregulation of lncRNA UCA1 could reduce oxidative stress and inflammation through the inhibition of the PI3K/Akt signaling pathway [46]. The central inflammation mechanism mediated by the excessive activation of microglia is also one of the important initiating factors for the development of PD [83]. The overexpressed lincRNA-p21 in PD regulates the activation of microglia through the feedback loop formed with the miR-181 family to promote the occurrence of neuroinflammation and the development of the disease [41].

\section{IncRNAs as Putative Biomarkers and Therapeutic Targets for PD}

Increasing scientific data show that certain lncRNAs alter differentially over time in the brains of patients with PD [48]. As the symptoms of Parkinson's disease improve, the level of lncRNA and microRNA also changes $[20,84]$. At the same time, a recent study showed that lnc-MKRN2-42: 1 in PD patients was positively correlated with MDS-UPDRS III score [52]. CSF is very close to the main site of PD pathology. As molecular changes in the PD patient's brain are reflected in CSF composition, the CSF represents an optimal source of biomarkers of PD. IncRNAs in CSF have the potential as a biomarker for PD because of the lack of a barrier between CSF and the brain. The changes in lncRNA expression levels in blood leukocyte samples may also be related to disease status [20]. It has been demonstrated by researchers that blood leukocytes can serve as a feasible and reliable tissue source to test for disease-induced and treatment-related transcript changes [85]. lncRNAs have a higher abundance than protein-coding genes, so more regulation of lncRNA expression can be observed in the same sample, which provides a greater possibility for examining lncRNA-based biomarkers. Large sizes of lncRNAs can fold into complex secondary/tertiary structures and scaffolds, through which they may interact with various proteins, transcriptional regulators, mRNA, and DNA sequences [86]. This may be associated with the initiation and progression of $\mathrm{PD}$. The existence of a large amount of regulation of lncRNA interaction sites for development based on the structure of the new PD drugs provides a broader platform. In addition, considering that lncRNA is involved in multiple cell signal transduction pathways in $\mathrm{PD}$, it can be used to formulate specific PD diagnosis and targeted therapy strategies. The potential mechanisms of action of lncRNAs include the inhibition of the expression of PD-linked genes, reduction in the production of alpha-synuclein, maintenance of autophagy system balance, delay in the apoptosis of dopaminergic neurons, alleviation of nerve inflammation, and so forth. All these findings indicate that lncRNAs have the potential to become a putative biomarker for PD. IncRNAs are expected to play an important role as a biomarker and therapeutic target for the early detection of PD.

Although the research of lncRNA as a biomarker in PD is still in its infancy, it is exciting that, in certain research areas, clinical trials have started on lncRNA as a biomarker [87-90]. In addition, circulating HOTAIR can be used in the diagnosis of breast cancer [91]. HOTAIR can be used to predict the recurrence of HCC [92]. MALAT1, UCA1, ANRIL, and NEAT1 can be used to predict early and metastatic lung cancer [93]. UCA1, H19, and HOTAIR can be used as biomarkers to detect bladder cancer [94]. All these indicate that lncRNA also has the potential to become a new diagnostic and prognostic marker for PD.

As the expression level of lncRNA in PD is related to the initiation and progression of PD and its symptoms, it can be used as a potential therapeutic target for PD. It is possible to target lncRNA to regulate its expression in a variety of ways. For example, the use of lncRNA-specific siRNA, such as the downregulation of siRNA-mediated MALAT1 expression can inhibit $\mathrm{MPP}(+)$-induced apoptosis of DA neurons [33]. In general, the therapy based on lncRNA as a biomarker and possible therapeutic target for PD is still promising.

\section{Conclusions}

The research on lncRNAs is in the initial stages. An increasing number of studies have been conducted on the role of lncRNAs in PD in the last three years. Some studies have shown that lncRNAs are involved in the initiation and progression of PD. A large number of lncRNAs have been found to provide a new basis for the development of early diagnosis and treatment of PD, and the expression of lncRNAs can also be used to predict the symptoms of PD patients. Previous studies have found that some lncRNAs 
play a protective role in PD (such as UCHL1, MAPT-AS1, and Mirt2), and some of them aggravate the disease progression (such as HOTAIR, MALAT1, NEAT1, lincrna-p21, and SNHG1). Now, many challenges in the study of lncRNAs cannot be ignored. For example, lncRNAs do not have a uniform nomenclature. Compared with coding genes, lncRNAs account for a small proportion, and it is difficult to determine the role of lncRNAs according to nucleotide sequences [95]. Research on lncRNA in PD is predicted to gain popularity in the future. Although researchers have identified some lncRNAs involved in PD using next-generation sequencing, the molecular mechanism of action of these lncRNAs still needs further verification. The sequencing technology is expensive. Moreover, the existing database is not sufficient to quantify the role of lncRNAs in PD. To verify the potential of lncRNAs in PD diagnosis and therapy, it is important to characterize each lncRNA in detail, such as the structure and function of each lncRNA, and to quantify the role of IncRNA in PD in multicenter studies. In the foreseeable future, early screening for PD may be more accurate, thanks to the studies on the lncRNA mechanism, thus improving the efficacy and accuracy of treatment.

\section{Conflicts of Interest}

The authors declare that they have no conflicts of interest regarding the publication of this article.

\section{Authors' Contributions}

Qiankun Lv wrote the manuscript, Ziyu Wang contributed to figure generation, Zhen Zhong contributed to table generation, and Wei Huang was involved in the project design, supervision, and manuscript revision. All authors read and approved the final manuscript.

\section{Acknowledgments}

The authors acknowledge the support and help from Professor Huang. They thank MedSci for linguistic assistance during the preparation of this manuscript. This study was funded by the Key Research and Development Project of Jiangxi Province (grant no. 20171BBG70065).

\section{References}

[1] L. V. Kalia and A. E. Lang, "Parkinson's disease," The Lancet, vol. 386, no. 9996, pp. 896-912, 2015.

[2] W. Poewe, K. Seppi, C. M. Tanner et al., "Parkinson disease," Nature Reviews Disease Primers, vol. 3, p. 17013, 2017.

[3] M. S. Hipp, P. Kasturi, and F. U. Hartl, "The proteostasis network and its decline in ageing," Nature Reviews Molecular Cell Biology, vol. 20, no. 7, pp. 421-435, 2019.

[4] S. K. Van Den Eeden, C. M. Tanner, A. L. Bernstein et al., "Incidence of parkinson's disease: variation by age, gender, and race/ethnicity," American Journal of Epidemiology, vol. 157, no. 11, pp. 1015-1022, 2003.

[5] D. Lin, Y. Liang, X. Jing et al., "Microarray analysis of an synthetic $\alpha$-synuclein induced cellular model reveals the expression profile of long non-coding RNA in parkinson's disease," Brain Research, vol. 1678, pp. 384-396, 2018.

[6] P. P. Michel, E. C. Hirsch, and S. Hunot, "Understanding dopaminergic cell death pathways in parkinson disease," Neuron, vol. 90, no. 4, pp. 675-691, 2016.

[7] A. Elkouzi, V. Vedam-Mai, R. S. Eisinger, and M. S. Okun, "Emerging therapies in parkinson disease-repurposed drugs and new approaches," Nature Reviews Neurology, vol. 15, no. 4, pp. 204-223, 2019.

[8] A. Capurro, L. G. Bodea, P. Schaefer, R. Luthi-Carter, and V. M. Perreau, "Computational deconvolution of genome wide expression data from parkinson's and huntington's disease brain tissues using population-specific expression analysis," Frontiers in Neuroscience, vol. 8, p. 441, 2014.

[9] W. Ren and X. Yang, "Pathophysiology of long non-coding RNAs in ischemic stroke," Frontiers in Molecular Neuroscience, vol. 11, p. 96, 2018.

[10] A. Fatica and I. Bozzoni, "Long non-coding RNAs: new players in cell differentiation and development," Nature Reviews Genetics, vol. 15, no. 1, pp. 7-21, 2014.

[11] T. R. Mercer, M. E. Dinger, and J. S. Mattick, "Long noncoding RNAs: insights into functions," Nature Reviews Genetics, vol. 10, no. 3, pp. 155-159, 2009.

[12] D. Bhartiya, S. Kapoor, S. Jalali et al., "Conceptual approaches for lncRNA drug discovery and future strategies," Expert Opinion on Drug Discovery, vol. 7, no. 6, pp. 503-513, 2012.

[13] Y. Zhang, X. Liu, X. Bai et al., "Melatonin prevents endothelial cell pyroptosis via regulation of long noncoding RNA MEG3/ miR-223/NLRP3 axis," Journal of Pineal Research, vol. 64, no. 2, 2018.

[14] H. J. Park, P. Ji, S. Kim et al., " 3 ' UTR shortening represses tumor-suppressor genes in trans by disrupting ceRNA crosstalk," Nature Genetics, vol. 50, no. 6, pp. 783-789, 2018.

[15] Z. Cheng, C. Yu, S. Cui et al., "circTP63 functions as a ceRNA to promote lung squamous cell carcinoma progression by upregulating FOXM1," Nature Communications, vol. 10, no. 1, p. 3200, 2019.

[16] B. Lee, A. Sahoo, J. Marchica et al., "The long noncoding RNA acts as an intranuclear organizing hub for pre-mRNA molecules," Science Advances, vol. 3, no. 5, 2017.

[17] O. Khorkova, J. Hsiao, and C. Wahlestedt, "Basic biology and therapeutic implications of lncRNA," Advanced Drug Delivery Reviews, vol. 87, pp. 15-24, 2015.

[18] J. L. Rinn and H. Y. Chang, "Genome regulation by long noncoding RNAs," Annual Review of Biochemistry, vol. 81, no. 1, pp. 145-166, 2012.

[19] X. Cao, G. Yeo, A. R. Muotri, T. Kuwabara, and F. H. Gage, "Noncoding RNAs in the mammalian central nervous system," Annual Review of Neuroscience, vol. 29, no. 1, pp. 77103, 2006.

[20] L. Soreq, A. Guffanti, N. Salomonis et al., "Long non-coding RNA and alternative splicing modulations in Parkinson's leukocytes identified by RNA sequencing," PLoS Computational Biology, vol. 10, no. 3, Article ID e1003517, 2014.

[21] R. Johnson, "Long non-coding RNAs in huntington's disease neurodegeneration," Neurobiology of Disease, vol. 46, no. 2, pp. 245-254, 2012.

[22] E. Mus, P. R. Hof, and H. Tiedge, "Dendritic BC200 RNA in aging and in alzheimer's disease," Proceedings of the National Academy of Sciences, vol. 104, no. 25, pp. 10679-10684, 2007.

[23] S. Narayan, K. Nakajima, and A. Sawa, "Disc1," The Neuroscientist, vol. 19, no. 5, pp. 451-464, 2013. 
[24] S. Chakrabarti and K. P. Mohanakumar, "Aging and neurodegeneration: a tangle of models and mechanisms," Aging and Disease, vol. 7, no. 2, pp. 111-113, 2016.

[25] A. R. Ghanam, Q. Xu, S. Ke, M. Azhar, Q. Cheng, and X. Song, "Shining the light on senescence associated LncRNAs," Aging and Disease, vol. 8, no. 2, pp. 149-161, 2017.

[26] T. R. Mercer, M. E. Dinger, S. M. Sunkin, M. F. Mehler, and J. S. Mattick, "Specific expression of long noncoding RNAs in the mouse brain," Proceedings of the National Academy of Sciences, vol. 105, no. 2, pp. 716-721, 2008.

[27] M. Majidinia, A. Mihanfar, R. Rahbarghazi, A. Nourazarian, B. Bagca, and Ç. B. Avci, "The roles of non-coding RNAs in parkinson's disease," Molecular Biology Reports, vol. 43, no. 11, pp. 1193-1204, 2016.

[28] C. Carrieri, A. R. Forrest, C. Santoro et al., "Expression analysis of the long non-coding RNA antisense to Uchl1 (AS Uchl1) during dopaminergic cells' differentiation in vitro and in neurochemical models of parkinson's disease," Frontiers in Cellular Neuroscience, vol. 9, p. 114, 2015.

[29] T. Peng, X. Liu, J. Wang et al., "Long noncoding RNA HAGLROS regulates apoptosis and autophagy in parkinson's disease via regulating miR-100/ATG10 axis and PI3K/Akt/ mTOR pathway activation," Artificial Cells, Nanomedicine, and Biotechnology, vol. 47, no. 1, pp. 2764-2774, 2019.

[30] S. Wang, X. Zhang, Y. Guo, H. Rong, and T. Liu, “The long noncoding RNA HOTAIR promotes parkinson's disease by upregulating LRRK2 expression," Oncotarget, vol. 8, no. 15, pp. 24449-24456, 2017.

[31] W. Liu, Q. Zhang, J. Zhang, W. Pan, J. Zhao, and Y. Xu, "Long non-coding RNA MALAT1 contributes to cell apoptosis by sponging miR-124 in parkinson disease," Cell \& Bioscience.vol. 7, p. 19, 2017.

[32] Q.-S. Zhang, Z.-H. Wang, J.-L. Zhang, Y.-L. Duan, G.-F. Li, and D.-L. Zheng, "Beta-asarone protects against MPTP-induced parkinson's disease via regulating long non-coding RNA MALAT1 and inhibiting $\alpha$-synuclein protein expression," Biomedicine \& Pharmacotherapy, vol. 83, pp. 153-159, 2016.

[33] Q. Chen, X. Huang, and R. Li, "IncRNA MALAT1/miR-205$5 \mathrm{p}$ axis regulates MPP-induced cell apoptosis in MN9D cells by directly targeting LRRK2," American Journal of Translational Research, vol. 10, no. 2, pp. 563-572, 2018.

[34] Y. Lu, Z. Gong, X. Jin, P. Zhao, Y. Zhang, and Z. Wang, "LncRNA MALAT1 targeting miR-124-3p regulates DAPK1 expression contributes to cell apoptosis in parkinson's disease," Journal of Cellular Biochemistry, 2020.

[35] K. G. Coupland, W. S. Kim, G. M. Halliday, M. Hallupp, C. Dobson-Stone, and J. B. Kwok, "Role of the long noncoding RNA MAPT-AS1 in regulation of microtubule associated protein tau (MAPT) expression in parkinson's disease," PLoS One, vol. 11, no. 6, Article ID e0157924, 2016.

[36] Y. Han, C. Kang, M. Kang, W. Quan, H. Gao, and Z. Zhong, "Long non-coding RNA Mirt2 prevents TNF- $\alpha$-triggered inflammation via the repression of microRNA-101," International Immunopharmacology, vol. 76, p. 105878, 2019.

[37] Y. Liu and Z. Lu, "Long non-coding RNA NEAT1 mediates the toxic of Parkinson's disease induced by MPTP/MPP+ via regulation of gene expression," Clinical and Experimental Pharmacology and Physiology, vol. 45, no. 8, pp. 841-848, 2018.

[38] W. Yan, Z.-Y. Chen, J.-Q. Chen, and H.-M. Chen, "LncRNA NEAT1 promotes autophagy in MPTP-induced parkinson's disease through stabilizing PINK1 protein," Biochemical and
Biophysical Research Communications, vol. 496, no. 4, pp. 1019-1024, 2018.

[39] X.-M. Ding, L.-J. Zhao, H.-Y. Qiao, S.-L. Wu, and X.-H. Wang, "Long non-coding RNA-p21 regulates $\mathrm{MPP} \pm$ induced neuronal injury by targeting miR-625 and derepressing TRPM2 in SH-SY5Y cells," Chemico-biological Interactions, vol. 307, pp. 73-81, 2019.

[40] X. Xu, C. Zhuang, Z. Wu, H. Qiu, H. Feng, and J. Wu, "LincRNA-p21 inhibits cell viability and promotes cell apoptosis in parkinson's disease through activating $\alpha$-synuclein expression," BioMed Research International, vol. 2018, Article ID 8181374, 10 pages, 2018.

[41] Y. Ye, X. He, F. Lu et al., "A lincRNA-p21/miR-181 family feedback loop regulates microglial activation during systemic LPS- and MPTP- induced neuroinflammation," Cell Death \& Disease, vol. 9, no. 8, p. 803, 2018.

[42] Y. Chen, Y.-j. Lian, Y.-q. Ma, C.-j. Wu, Y.-k. Zheng, and N.-c. Xie, "LncRNA SNHG1 promotes $\alpha$-synuclein aggregation and toxicity by targeting miR-15b-5p to activate SIAH1 in human neuroblastoma SH-SY5Y cells," Neurotoxicology, vol. 68, pp. 212-221, 2018.

[43] C. Qian, Y. Ye, H. Mao et al., "Downregulated IncRNASNHG1 enhances autophagy and prevents cell death through the miR-221/222/p27/mTOR pathway in parkinson's disease," Experimental Cell Research, vol. 384, no. 1, p. 111614, 2019.

[44] J. Zhao, L. Geng, Y. Chen, and C. Wu, "SNHG1 promotes MPP-induced cytotoxicity by regulating PTEN/AKT/mTOR signaling pathway in SH-SY5Y cells via sponging miR-1533p," Journal of Biological Research, vol. 53, no. 1, p. 1, 2020.

[45] Y. Zhang, Q. Xia, and J. Lin, "LncRNA H19 attenuates apoptosis in MPTP-induced parkinson's disease through regulating miR-585-3p/PIK3R3," Neurochemical Research, 2020.

[46] L. Cai, L. Tu, T. Li et al., "Downregulation of lncRNA UCA1 ameliorates the damage of dopaminergic neurons, reduces oxidative stress and inflammation in parkinson's disease through the inhibition of the PI3K/Akt signaling pathway," International Immunopharmacology, vol. 75, p. 105734, 2019.

[47] M. Elkouris, G. Kouroupi, A. Vourvoukelis et al., "Long noncoding RNAs associated with neurodegeneration-linked genes are reduced in parkinson's disease patients," Frontiers in Cellular Neuroscience, vol. 13, p. 58, 2019.

[48] T. F. J. Kraus, M. Haider, J. Spanner, M. Steinmaurer, V. Dietinger, and H. A. Kretzschmar, "Altered long noncoding RNA expression precedes the course of parkinson's disease-a preliminary report," Molecular Neurobiology, vol. 54, no. 4, pp. 2869-2877, 2017.

[49] Y. Gui, H. Liu, L. Zhang, W. Lv, and X. Hu, "Altered microRNA profiles in cerebrospinal fluid exosome in parkinson disease and alzheimer disease," Oncotarget, vol. 6, no. 35, pp. 37043-37053, 2015.

[50] A. Hossein-Nezhad, R. P. Fatemi, R. Ahmad et al., "Transcriptomic profiling of extracellular RNAs present in cerebrospinal fluid identifies differentially expressed transcripts in parkinson's disease," Journal of Parkinson's Disease, vol. 6, no. 1, pp. 109-117, 2016.

[51] Y. Ni, H. Huang, Y. Chen, M. Cao, H. Zhou, and Y. Zhang, "Investigation of long non-coding RNA expression profiles in the substantia nigra of parkinson's disease," Cellular and Molecular Neurobiology, vol. 37, no. 2, pp. 329-338, 2017.

[52] Q. Wang, C.-L. Han, K.-L. Wang et al., "Integrated analysis of exosomal lncRNA and mRNA expression profiles reveals the involvement of lnc-MKRN2-42:1 in the pathogenesis of parkinson's disease," CNS Neuroscience \& Therapeutics, vol. 26, 2019. 
[53] F. Jiao, Q. Wang, P. Zhang, L. Bu, J. Yan, and B. Tian, "Expression signatures of long non-coding RNA in the substantia nigra of pre-symptomatic mouse model of parkinson's disease," Behavioural Brain Research, vol. 331, pp. 123-130, 2017.

[54] J. Liu, Y. Xu, Y. Kang et al., “The aberrantly expressed long non-coding RNA in the substantia nigra and corpus striatum of Nrf2-knockout mice," Journal of Neurochemistry, vol. 143, no. 1, pp. 65-75, 2017.

[55] X.-z. Li, S.-n. Zhang, F. Lu, and S.-m. Liu, "Microarray expression analysis for the paradoxical roles of acanthopanax senticosusHarms in treating $\alpha$-synucleinopathies," Phytotherapy Research, vol. 30, no. 2, pp. 243-252, 2016.

[56] J. Li, Y. Sun, and J. Chen, "Transcriptome sequencing in a 6hydroxydopamine rat model of parkinson's disease," Genes \& Genetic Systems, vol. 94, no. 2, pp. 61-69, 2019.

[57] L. Parnetti, A. Castrioto, D. Chiasserini et al., "Cerebrospinal fluid biomarkers in parkinson disease," Nature Reviews Neurology, vol. 9, no. 3, pp. 131-140, 2013.

[58] T. L. Edwards, W. K. Scott, C. Almonte et al., "Genome-wide association study confirms SNPs in SNCAand the MAPTRegion as common risk factors for parkinson disease," Annals of Human Genetics, vol. 74, no. 2, pp. 97-109, 2010.

[59] S. J. Adams, R. J. P. Crook, M. Deture et al., "Overexpression of wild-type murine tau results in progressive tauopathy and neurodegeneration," The American Journal of Pathology, vol. 175, no. 4, pp. 1598-1609, 2009.

[60] K. G. Coupland, G. D. Mellick, P. A. Silburn et al., "DNA methylation of the MAPT gene in parkinson's disease cohorts and modulation by vitamin E in vitro," Movement Disorders, vol. 29, no. 13, pp. 1606-1614, 2014.

[61] J. L. Robinson, E. B. Lee, S. X. Xie et al., "Neurodegenerative disease concomitant proteinopathies are prevalent, age-related and APOE4-associated," Brain, vol. 141, no. 7, pp. 2181-2193, 2018.

[62] N. Bengoa-Vergniory, R. F. Roberts, R. Wade-Martins, and J. Alegre-Abarrategui, "Alpha-synuclein oligomers: a new hope," Acta Neuropathologica, vol. 134, no. 6, pp. 819-838, 2017.

[63] B. Dehay, M. Bourdenx, P. Gorry et al., "Targeting $\alpha$-synuclein for treatment of parkinson's disease: mechanistic and therapeutic considerations," The Lancet Neurology, vol. 14, no. 8, pp. 855-866, 2015.

[64] D. Bernard, K. V. Prasanth, V. Tripathi et al., "A long nuclearretained non-coding RNA regulates synaptogenesis by modulating gene expression," The EMBO Journal, vol. 29, no. 18, pp. 3082-3093, 2010.

[65] L. Lipovich, F. Dachet, J. Cai et al., "Activity-dependent human brain coding/noncoding gene regulatory networks," Genetics, vol. 192, no. 3, pp. 1133-1148, 2012.

[66] H. A. Lashuel, C. R. Overk, A. Oueslati, and E. Masliah, "The many faces of $\alpha$-synuclein: from structure and toxicity to therapeutic target," Nature Reviews Neuroscience, vol. 14, no. 1, pp. 38-48, 2013.

[67] M. Xilouri, O. R. Brekk, and L. Stefanis, "Alpha-synuclein and protein degradation systems: a reciprocal relationship," Molecular Neurobiology, vol. 47, no. 2, pp. 537-551, 2013.

[68] Y. Hou, X. Dan, M. Babbar et al., "Ageing as a risk factor for neurodegenerative disease," Nature Reviews Neurology, vol. 2019, Article ID 8181374, 10 pages, 2019.

[69] S. Kaushik and A. M. Cuervo, "Proteostasis and aging," Nature Medicine, vol. 21, no. 12, pp. 1406-1415, 2015.

[70] P. Brundin, J.-Y. Li, J. L. Holton, O. Lindvall, and T. Revesz, "Research in motion: the enigma of parkinson's disease pathology spread," Nature Reviews Neuroscience, vol. 9, no. 10, pp. 741-745, 2008.

[71] M. A. Nalls, N. Pankratz, C. M. Lill et al., "Large-scale metaanalysis of genome-wide association data identifies six new risk loci for parkinson's disease," Nature Genetics, vol. 46, no. 9, pp. 989-993, 2014.

[72] F. Soldner, Y. Stelzer, C. S. Shivalila et al., "Parkinson-associated risk variant in distal enhancer of $\alpha$-synuclein modulates target gene expression," Nature, vol. 533, no. 7601, pp. 95-99, 2016.

[73] A. Garrido, I. Aldecoa, E. Gelpi, and E. Tolosa, “Aggregation of $\alpha$-synuclein in the gonadal tissue of 2 patients with parkinson disease," JAMA Neurology, vol. 74, no. 5, pp. 606-607, 2017.

[74] K. Mokretar, D. Pease, J.-W. Taanman et al., "Somatic copy number gains of $\alpha$-synuclein (SNCA) in parkinson's disease and multiple system atrophy brains," Brain, vol. 141, no. 8, pp. 2419-2431, 2018.

[75] G. E. Voutsinas, E. F. Stavrou, G. Karousos et al., "Allelic imbalance of expression and epigenetic regulation within the alpha-synuclein wild-type and p.Ala53Thr alleles in parkinson disease," Human Mutation, vol. 31, no. 6, pp. 685-691, 2010.

[76] M. Lu, W. L. Sun, J. Shen et al., "LncRNA-UCA1 promotes PD development by upregulating SNCA," European Review for Medical and Pharmacological Sciences, vol. 22, no. 22, pp. 7908-7915, 2018.

[77] M. B. Ramirez, J. Madero-Perez, P. Rivero-Rios et al., "LRRK2 and parkinson's disease: from lack of structure to gain of function," Current Protein \& Peptide Science, vol. 18, no. 7, pp. 677-686, 2017.

[78] N. Joshi and S. Singh, "Updates on immunity and inflammation in parkinson disease pathology," Journal of Neuroscience Research, vol. 96, no. 3, pp. 379-390, 2018.

[79] Y. Chao, S. C. Wong, and E. K. Tan, "Evidence of inflammatory system involvement in parkinson's disease," BioMed Research International, vol. 2014, Article ID 308654, 9 pages, 2014.

[80] Z. Chen and B. D. Trapp, "Microglia and neuroprotection," Journal of Neurochemistry, vol. 136, no. 1, pp. 10-17, 2016.

[81] M. Du, L. Yuan, X. Tan et al., "The LPS-inducible lncRNA Mirt2 is a negative regulator of inflammation," Nature Communications, vol. 8, no. 1, p. 2049, 2017.

[82] J. Luo, K. Wang, S. Yeh et al., "LncRNA-p21 alters the antiandrogen enzalutamide-induced prostate cancer neuroendocrine differentiation via modulating the EZH2/STAT3 signaling," Nature Communications, vol. 10, no. 1, p. 2571, 2019.

[83] Z. Fan, Y. Aman, I. Ahmed et al., "Influence of microglial activation on neuronal function in alzheimer's and parkinson's disease dementia," Alzheimer's \& Dementia, vol. 11, no. 6, pp. 608-621, 2015.

[84] L. Soreq, N. Salomonis, M. Bronstein et al., "Small RNA sequencing-microarray analyses in parkinson leukocytes reveal deep brain stimulation-induced splicing changes that classify brain region transcriptomes," Frontiers in Molecular Neuroscience, vol. 6, p. 10, 2013.

[85] L. Soreq, H. Bergman, Z. Israel, and H. Soreq, “Overlapping molecular signatures in parkinson's patients' leukocytes before and after treatment and in mouse model brain regions," CNS \&amp; Neurological Disorders Drug Targets, vol. 12, no. 8, pp. 1086-1093, 2013.

[86] A. Bhan, M. Soleimani, and S. S. Mandal, "Long noncoding RNA and cancer: a new paradigm," Cancer Research, vol. 77, no. 15, pp. 3965-3981, 2017. 
[87] Circulating Non-Coding RNA in Acute Ischemic Stroke, https://ClinicalTrials.gov/show/NCT04175691.

[88] Regulation of LncRNA for Breg in Patients with Thymoma and Autoimmune Diseases, https://ClinicalTrials.gov/show/ NCT02948855.

[89] Serum LncRNAs as Early Potential Biomarkers for the Prediction of Preeclampsia, https://ClinicalTrials.gov/show/ NCT03903393.

[90] TAEC-GP versus AEC-T for the High Risk TNBC Patients and Validation of the mRNA-lncRNA Signature, https:// ClinicalTrials.gov/show/NCT02641847.

[91] L. Zhang, X. Song, X. Wang et al., "Circulating DNA of HOTAIR in serum is a novel biomarker for breast cancer," Breast Cancer Research and Treatment, vol. 152, no. 1, pp. 199-208, 2015.

[92] Y. Geng, S. Xie, Q. Li, J. Ma, and G. Wang, "Large intervening non-coding RNA HOTAIR is associated with hepatocellular carcinoma progression," Journal of International Medical Research, vol. 39, no. 6, pp. 2119-2128, 2011.

[93] T. Gutschner, M. Hämmerle, M. Eissmann et al., "The noncoding RNA MALAT1 is a critical regulator of the metastasis phenotype of lung cancer cells," Cancer Research, vol. 73, no. 3, pp. 1180-1189, 2013.

[94] F. Wang, X. Li, X. Xie, L. Zhao, and W. Chen, "UCA1, a nonprotein-coding RNA up-regulated in bladder carcinoma and embryo, influencing cell growth and promoting invasion," FEBS Letters, vol. 582, no. 13, pp. 1919-1927, 2008.

[95] R. A. Chodroff, L. Goodstadt, T. M. Sirey et al., "Long noncoding RNA genes: conservation of sequence and brain expression among diverse amniotes," Genome Biology, vol. 11, no. 7, p. R72, 2010. 